\title{
The Mitral Valve Meeting in Zurich - a world upside down
}

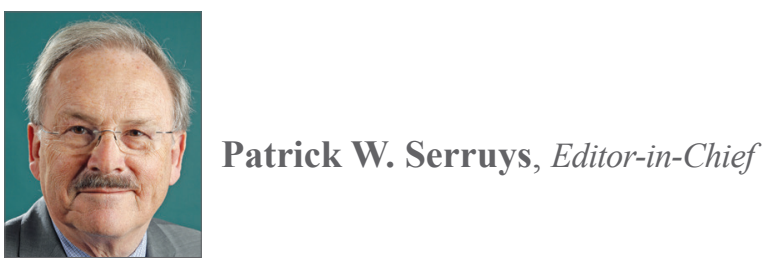

I recently had the pleasure of participating once again in the Mitral Valve Meeting in Zurich under the course directorship of Francesco Maisano and Marty Leon. This year's edition, the second, featured even more interaction and practical workshops whilst maintaining the strong contemporary Heart Team philosophy. What is fascinating about this meeting is the high, uppermost level of education for both the surgeon and the interventionalist. It is my assessment that the audience was split 50-50 between the two groups, which in itself is a remarkable achievement, and that furthermore the Heart Team approach clearly indicates that the two communities are now working side by side.

The first day consisted of four module sessions comprising eight different workshops for each module. All those who registered for the meeting had to participate in the workshops. As an example, let me describe one workshop in which I participated as faculty member. Our workshop team included Mark Reisman from Seattle, who is also well known as a fabulous teacher of anatomy (in particular, the anatomical relationships with devices), Alberto Weber a surgeon from the Zurich team, an echographist from Zurich and myself. Eight to ten tables were set up in the workshop room and on each table there was a pig's heart. In fact, in all, 150 pigs' hearts were dissected by the physicians attending the workshops. One may remark in jest that these animals not only feed our stomachs but also feed our brains with a new understanding of mitral interventions.

The instructor started to dissect the heart under angioscopy, with the images being projected on a large 3D screen. Each attendee had special 3D spectacles aiding a better perception of the projected images on the screen with which we systematically learned about the trigone of the mitral valve, the primary and secondary chordae, the basal chordae, and the insertion to the papillary muscle. For the whole workshop we had to dissect the heart in great detail. In between, there were short lectures on 3D echography, the anatomy and implantation of surgical devices, something which was also demonstrated live during the workshop.

I felt that all the attendees during this meeting may have reached the ultimate level of education. The other two days were spectacular in the sense that more than ever we realised the extraordinary privilege of the surgeon operating on a static heart, with a perfect view of the anatomy in terms of prolapse within the P2 segment. This is knowledge which the average interventionalist has never been exposed to in his career. Beyond this extraordinary asset of the surgeon, I realised that they, the surgeons, were working on a non-beating heart with a perfect view but were unable to judge by haemodynamics and motion what they had achieved by their surgery. Their most sophisticated assessment is the water test which consists of injecting a certain amount of water into the left ventricle to see if there is a leak or reflux of water into the left atrium. Following this wonderful case, Alberto Weber implanted two or three artificial chordae. This was then followed by Francesco Maisano's case, which had the totally opposite perception of a somewhat similar pathology.

During Francesco's case, it was the surgeon performing the percutaneous intervention - to coin a phrase, the world upside down. Amazingly, he implanted three clips on the mitral valve, while at the same time we could see the $\mathrm{V}$-wave reduce within 45 minutes from $40 \mathrm{mmHg}$ to $12 \mathrm{mmHg}$. This is in effect basically normalising the pressure in the left atrium and monitored, by the way, via a pigtail which ran parallel to the sheath for the clips. It is at this moment that one realises that the imaging is not a direct view but indirect, using 2D and 3D echo, and yet seeing with the beating heart the whole haemodynamic change during the treatment. This improvement was progressively achieved with the implantation of each clip serially implanted (in total three), with continuous assessment of the haemodynamics using 2D and 3D echo but also Doppler for flow and velocity assessment. The contrast is that, while the surgeon is a tailor working on something "static", the interventionalist is working "dynamically" such that the effect of his work can be judged immediately. And as a little footnote, after the 3 clip implantations, Francesco took advantage of having access to the left atrium to close the left appendage....as a good surgeon would do...one stone two birds.

Personally, I think that the perception of the future has never been so evident. I would not like to be tempted into making a prediction but, with 26 to 40 devices existing in the mitral space, devices which basically mimic the ingenious, dexterous and masterful surgeon over the last 30 to 40 years, this percutaneous approach is probably the way forward.

Moving on from the Zurich meeting, I am now eagerly looking forward to PCR Innovators Day in Paris in May where one of the four key topics during this meeting will be the latest innovations in the mitral and tricuspid valve interventional arena under the expert guidance of Nicolo Piazza and Michel Darnaud. And then, of course, the highlight of the year will be the world's largest educational meeting on valvular heart disease and percutaneous valve interventions - PCR London Valves in September in London. 